\title{
Cadherina E, Catenina Beta, Antígeno Ki-67 y proteína p53 en el pronóstico de la recidiva tumoral en los tumores superficiales de vejiga $\mathrm{T} 1$
}

\author{
Moyano Calvo JL, Blanco Palenciano E, Beato Moreno A*, Gutiérrez González M, \\ Pérez-Lanzac Lorca A, Samaniego Torres A, Montaño JA, Castiñeiras Fernández J.
}

Servicio de Urología. Hospital Virgen Macarena. *Departamento de la Facultad de Matemáticas. Sevilla.

Actas Urol Esp. 2006;30(9):871-878

\section{RESUMEN}

CADHERINA E, CATENINA BETA, ANTÍGENO Ki-67 Y PROTEÍNA P53 EN EL PRONÓSTICO DE LA RECIDIVA TUMORAL EN LOS TUMORES SUPERFICIALES DE VEJIGA T1

Introducción: La historia natural del carcinoma superficial de vejiga (CSV) se caracteriza por su alta tasa de recidivas y por la capacidad de progresar a estadios infiltrantes. Vamos a investigar la capacidad de predicción de recidiva tumoral de la proteína p53, el antígeno Ki-67 y las moléculas de adhesión celular Cadherina E y Catenina Beta

Material y método: 88 tumores T1 con una mediana de tiempo libre de enfermedad de 36 meses. Han recibido tratamiento profiláctico con BCG $81 \mathrm{mg}$. semanal durante seis semanas el 58\% de la serie. Para p53 y Ki-67 se estableció el nivel de $10 \%$ de células teñidas para considerar positivo el tumor. Para Cadherina E y Catenina se han establecido dos grupos: uno con los valores 0-4 (negativo), y otro con los valores 5-8 (positivo).

Resultados: Han recidivado el $31 \%$ de los tumores y progresado a estadio infiltrante el $3 \%$ La expresión de Ki-67 se correlaciona con grado ( $p, 002)$ y permeación linfática $(\mathrm{p}, 028)$. La multiplicidad tumoral con la falta de expresión de Cadherina E y Catenina Beta. Sólo la expresión de Ki-67 (p .049) y la de Catenina Beta (p .039) alcanzan significación estadística. En el estudio multivariante sólo la falta de expresión de Catenina Beta muestra tener valor pronóstico independiente para recidiva (p .049; O.R : 2,4-6,9)

Conclusiones: Los marcadores más útiles son Ki-67 y Catenina Beta. Sólo la Catenina Beta muestra un valor independiente para recidiva tumoral.

Los tumores que no expresan Catenina Beta o Cadherina E tienen un menor tiempo libre de recidiva.

Palabras clave: Factores pronósticos. Carcinoma superficial de vejiga. Inmunohistoquimica.

\section{ABSTRACT}

PROGNOSTIC VALUE OF E-CADHERINA, BETA CATENIN, KI, Ki-67 ANTIGEN AND P53 PROTEIN IN THE SUPERFICIAL BLADDER TUMORS

Introduction: The natural history of the superficial carcinoma of bladder is characterized by his high rate of recurrence and by the aptitude to progress to higher stages. We are going to investigate the capacity of prediction for tumor recurrence of protein p53, antigen Ki-67, E Cadherin and Beta Catenin

Material and method: $88 \mathrm{~T} 1$ tumors with a median of free time of disease of 36 months. $58 \%$ of the serie has received prophylactic treatment with BCG $81 \mathrm{mg}$. weekly for six weeks. Cut-oof level for For P53 and Ki-67 is 10 $\%$ of stained cells. For E Cadherin and Beta Catenin we have established two groups: one with the values 0-4 (negative), and other one with the values 5-8 (positive).

Results: Recurrence rate $31 \%$, stage progression $3 \%$. Ki-67 expression is correlated with grade (p .002) and lymphatic permeation (p .028). Multiplicity is correlated with lack( of Cadherin and Catenin's expression. Only Ki-67 expression (p .049) and lack of Beta Catenin expression (p .039) reach statistical significance. In multivariant study only lack of Beta Catenin's expression shows independent recurrence value (p .049; O.R: 2,4-6,9)

Conclusions: The most useful prognmostic markers are Ki-67 and Catenina Beta

Only Beta Catenin Beta shows independent value for tumour recurrence.

Tumors wich lack expression for Catenin B or Cadherin E have lower recurrence free time. 
L a historia natural del carcinoma superficial de vejiga (CSV) se caracteriza por su alta tasa de recidiva y por la capacidad de progresar a estadios infiltrantes. Las características histopatológicas “clásicas" del tumor (estadio, grado, permeación linfática, número, tamaño...) no tienen en muchos casos la suficiente capacidad para predecir la evolución y el potencial agresivo del tumor. La llegada de la biología molecular al campo de la oncología vesical ha permitido la aplicación de la misma para intentar mejorar la predicción del pronóstico de los TSV.

En este trabajo, vamos a investigar de forma retrospectiva la capacidad de predecir la recidiva tumoral de la proteína p53, el antígeno Ki-67 y las moléculas de adhesión celular Cadherina $\mathrm{E}$ y Catenina Beta

La proteína p53 es un regulador de expresión génica que actúa como factor de trascripción capaz de activar y reprimir determinadas proteínas que actúan en el ciclo celular en el paso de G1 a S.

La forma nativa o "wild" tiene una vida media corta, inferior a veinte minutos, por lo que es muy difícil su detección por técnicas de inmunohistoquímica. Sin embargo, la mutación del gen lleva a la expresión de formas mutadas de proteína que en la mayoría de las ocasiones prolonga su vida media, facilitando su determinación.

El valor pronóstico de la proteína p53 en el carcinoma superficial está por definir. Así, hay autores que defienden su capacidad de predecir la recidiva ${ }^{1,2}$ y progresión ${ }^{4-6}$ incluso en estadios $\mathrm{Ta}^{2,7}$, mientras que otros no encuentran tal capacidad pronóstica ni para recidiva ${ }^{7-10}$, ni para progresión ${ }^{8,11,12}$.

Esta discrepancia de resultados puede deberse a:

- Diversidad de anticuerpos (DO-7, pAb 1801.) con distintos resultados en el mismo grupo de enfermos ${ }^{13}$.

- Distintos niveles de corte.

- Problemas metodológicos tales como número de enfermos, escaso seguimiento, procesamiento de la muestra.

- Algunas mutaciones del gen no prolongan la vida media de la proteína y no son detectables por inmunohistoquímica.
- Ausencia de relación causal entre mutación y acumulación de proteína ${ }^{14}$.

Dentro de España, la situación es similar, con autores que consideran la determinación de p53 útil para predecir recidiva ${ }^{7,15,16}$ o progresión ${ }^{15,17,18}$ mientras que otros no encuentran que aporte nada en recidiva ${ }^{17-20}$ ni en progresión ${ }^{19-21}$.

La Cadherina $\mathrm{E}$ forma parte de un conjunto de moléculas, llamadas de adherencia celular, que participan activamente en las interacciones de célula a célula y las interacciones entre células y componentes de la matriz extracelular.

Las Cadherinas son una familia de glicoproteínas transmembrana que participan en la adherencia celular calcio dependiente. Se fijan entre sí mediante interacciones homotípicas homofílicas y son cruciales para establecer y mantener las conexiones intercelulares y controlar la polaridad y morfología tisular.

Tienen una región extracelular, una región transmembrana y una región carboxi-terminal localizada en el citoplasma. Esta porción se une al citoesqueleto celular a través de otras proteínas llamadas Cateninas (alfa, beta y gamma). Esta asociación es esencial para que las Cadherinas ejerzan correctamente su función.

Se dividen en más de veinte subclases siendo la Cadherina $\mathrm{E}$ la correspondiente al epitelio, aunque no de manera exclusiva.

La Cadherina E es codificada en el brazo largo del cromosoma 16, en la posición 22.1. Se ha pensado que el gen de la Cadherina E sería un gen supresor ya que existe relación entre mutaciones a ese nivel y pérdida de expresión de Cadherina $\mathrm{E}$ en las tinciones tisulares.

La pérdida de cohesión de las células tumorales es uno de los procesos principales en la invasión y metástasis tumoral. La Cadherina E juega un papel importante en este proceso ya que la Cadherina $\mathrm{E}$ juega un papel fundamental para mantener la integridad y la polaridad del epitelio y su pérdida o disminución favorece la dispersión de las células tumorales.

En el urotelio normal su expresión es homogénea en los bordes celulares excepto en el que se encuentra en contacto con la membrana basal

En los tumores de vejiga se ha observado una disminución de su expresión con aumento del grado y del estadio $22-24$. 
Se ha asociado a recidiva tumoral ${ }^{25,26}$. Sin embargo, donde la mayoría de los autores han observado que su falta de expresión se relaciona con progresión tumoral ${ }^{22,26}$ y un tiempo de supervivencia corto $22,23,26,27$. Incluso dentro de los tumores infiltrantes permite establecer distintos nivele de pronóstico según el grado de tinción ${ }^{22}$.

Las Cateninas son proteínas intracistoplasmáticas que unen el extremo carboxi-terminal de las Cadherinas al citoesqueleto celular de actina. Se distinguen tres tipos: alfa, beta y gamma.

En el urotelio sano se localizan en los bordes celulares pero no el lado que se apoya en la membrana basal.

Es escasa la literatura que investiga el papel de las Cateninas en el cáncer de vejiga. De ellas la más estudiada has sido la Catenina Beta. La pérdida de su expresión se relaciona con mal pronóstico y corta supervivencia pero siempre asociada a la pérdida de expresión de Cadherina E. Por si sola no tiene valor pronóstico independiente para predecir supervivencia del enfermo ${ }^{22,27}$. Así mismo su expresión disminuye con el grado y el estadio ${ }^{24,28}$.

La proteína Ki-67 se encuentra presente en el núcleo de las células en proliferación celular (aumentando su concentración desde la fase G1 a la fase M, en la cual su nivel es el más alto), pero no en las que se encuentran en fase quiescente o en reposo (G0).

Las alteraciones en la proliferación celular, que surgen de la pérdida de control del ciclo celular, pueden ser estudiadas y cuantificadas determinando la expresión nuclear de proteína Ki-67 mediante técnicas de inmunohistoquímica.

La expresión de Ki-67 se correlaciona bien con el grado y estadio tumoral ${ }^{4,29,30}$.

Cada vez existen más estudios mostrando en el análisis multivariado que la expresión de Ki-67 es un marcador pronóstico independiente de recidiva $^{3,9,10,22,28,49-54}$ como de progresión ${ }^{31-33}$, si bien algunos autores no encuentran que su determinación aporte nada ni en la recidiva tumoral $^{4,8,33}$ ni en la progresión ${ }^{4,5,8}$.

En los tumores de grado bajo e intermedio (G1-G2) considerados de bajo riesgo, la determinación de Ki-67 permite diferenciar grupos de mayor agresividad, que justificaría en ellos una terapia más agresiva que la que le correspondería por sus características de estadio y grado ${ }^{6,31}$. Incluso en aquellos tumores únicos y bien diferenciados, la expresión de Ki-67 nos indica aquellos con mayor probabilidad de recurrir antes del año ${ }^{34}$.

En España son pocos los autores que han estudiado la relación existente entre Ki-67 y la recidiva y progresión tumoral en los tumores de vejiga. Rodríguez Alonso ${ }^{18}$, San Miguel Fraile ${ }^{16}$, Moyano Calvo ${ }^{17}$ y López Beltrán ${ }^{35}$ observan que la expresión de Ki-67 tiene valor pronóstico para recidiva tumoral, mientras que para progresión tumoral sólo la encuentra Rodríguez Alonso cuando establece el nivel de corte al $27 \%$.

\section{MATERIAL Y MÉTODO}

Hemos determinado, además de las variables clásicas, la expresión de Cadherina $\mathrm{E}$, Catenina Beta, antígeno Ki-67 y proteína p53 en 88 carcinomas superficiales de vejiga que reunían los siguientes requisitos:

- RTU completa

- Localización exclusivamente vesical

- Ausencia de Tis asociado

- Seguimiento mínimo de dos años.

Los estudios inmunohistoquímicos para la determinación de la expresión de antígeno Ki-67, proteína p53 , Cadherina E y Catenina Beta se han realizado aplicando la técnica inmunoperoxidásica, método $\mathrm{ABC}$ (complejo streptavidina-biotinaperoxidasa) para material incluido en parafina.

$\mathrm{El}$ desenmascaramiento del antígeno se realizó mediante calor en olla Duramatic (Kunhrikon) con tampón citrato al 1:100 manteniendo durante dos minutos a máxima temperatura.

- Para identificar el antígeno Ki 67 se utilizó el anticuerpo monoclonal IgG1 anti-Ki-67 MIB-1 (Byosistems SA, Costa Brava 30, Barcelona) durante 32 minutos.

- Para identificar la proteína p53 se utilizó el anticuerpo monoclonal IgG2a kappa anti-p53 BP53-12 (Byosistems SA, Costa Brava 30, Barcelona) durante el mismo periodo de tiempo.

- Para identificar la Cadherina E se utilizó el anticuerpo monoclonal Novocastra NCL-E-Cad a dilución 1:200 durante 18-24 horas.

- Para identificar la Catenina Beta se utilizó el anticuerpo monoclonal Novocastra NCL-B-Cat a dilución 1:25 durante 18-24 horas. 
Para determinar la proporción de Ki-67 y p53 se ha procedido a contar el número de células positivas en 10 campos de gran aumento $(\mathrm{x} 400)$ y dividirla por el número de células existentes en ese campo. Para determinar el número total de células existente por campo se ha procedido a contar las mismas mediante morfómetro por el mismo observador.

En ambos casos se estableció el nivel de 10\% de células teñidas para considerar positivo el tumor (Figs. 1 y 2).

La valoración de la expresión de Cadherina $\mathrm{E}$ y Catenina Beta se realizó midiendo la extensión y la intensidad de la tinción de 0 a 4 y de manera independiente, según siguiente esquema:
Extensión

0 no se observa tinción

1 tinción menor del 25\%

2 tinción entre el $25 \%$ y el $50 \%$

3 tinción entre el 50\% y el 75\%

4 tinción superior al 75\%

Intensidad

0 ausencia de tinción

1 tinción débil

2 tinción débil-moderada

3 tinción moderada-intensa

4 tinción intensa

En ambos casos se han establecido dos grupos: uno con los valores 0-4 (negativo), y otro con los valores 5-8 (positivo) (Figs. 3 y 4).

Para garantizar la fiabilidad de la técnica, en cada serie de tinción se realizó un control positivo previamente contrastado así como un control negativo en el que se sustituyó el anticuerpo primario específico por suero normal.

Para la variable Tamaño se han establecido dos grupos, uno con tamaño menor que 3 cc y otro con mayor o igual a $3 \mathrm{cc}$, y el número se ha agrupado en único y múltiple.

Los tumores T1G2 y T1G3 fueron tratados mediante instilación intravesical con $81 \mathrm{mg}$ de BCG Connaught semanalmente durante seis semanas, comenzado las instilaciones entre las tres y cuatro semanas posteriores a la resección. El seguimiento se ha efectuado mediante ecografia y citología. Cuatrimestralmente durante los dos primeros años, semestralmente los tres siguientes y anualmente desde entonces. Siempre que se ha considerado necesario se ha realizado cistoscopia.

En el análisis estadístico se ha utilizado la prueba Chi cuadrado para las variables discretas. $\mathrm{El}$ análisis multivariante se ha realizado siguiendo el método de regresión logística multivariante de Cox. El estudio de supervivencia libre de enfermedad se ha realizado mediante el método de Kaplan-Meyer.

FIGURA 2. Inmunotinción para p53. (x400). 


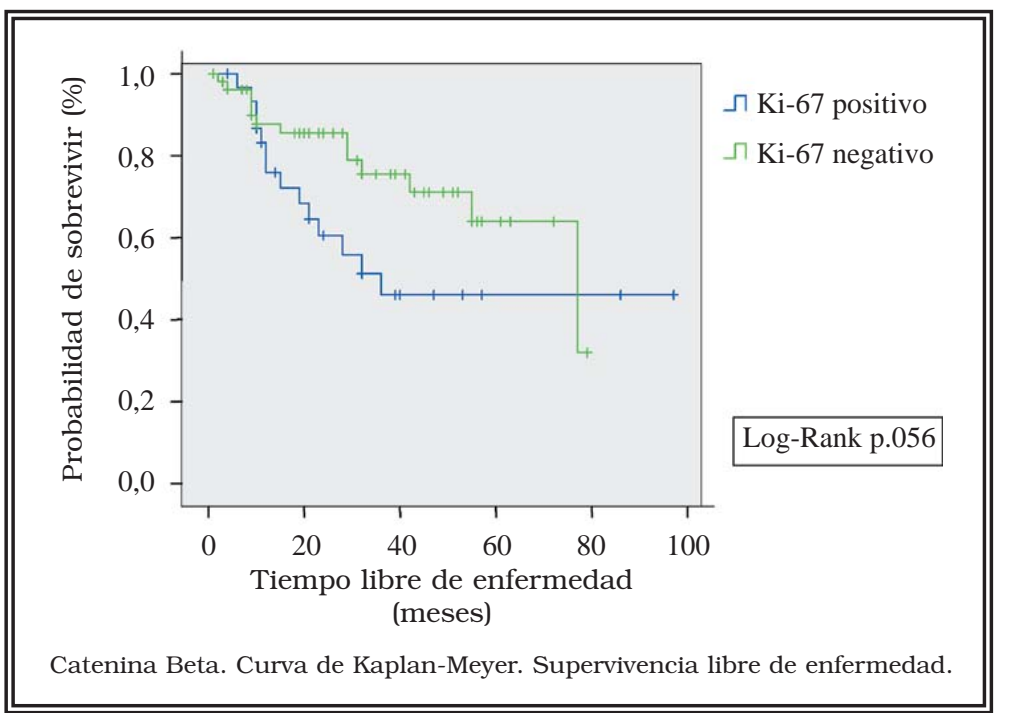

FIGURA 3. Inmunotinción para Catenina Beta. (x400).

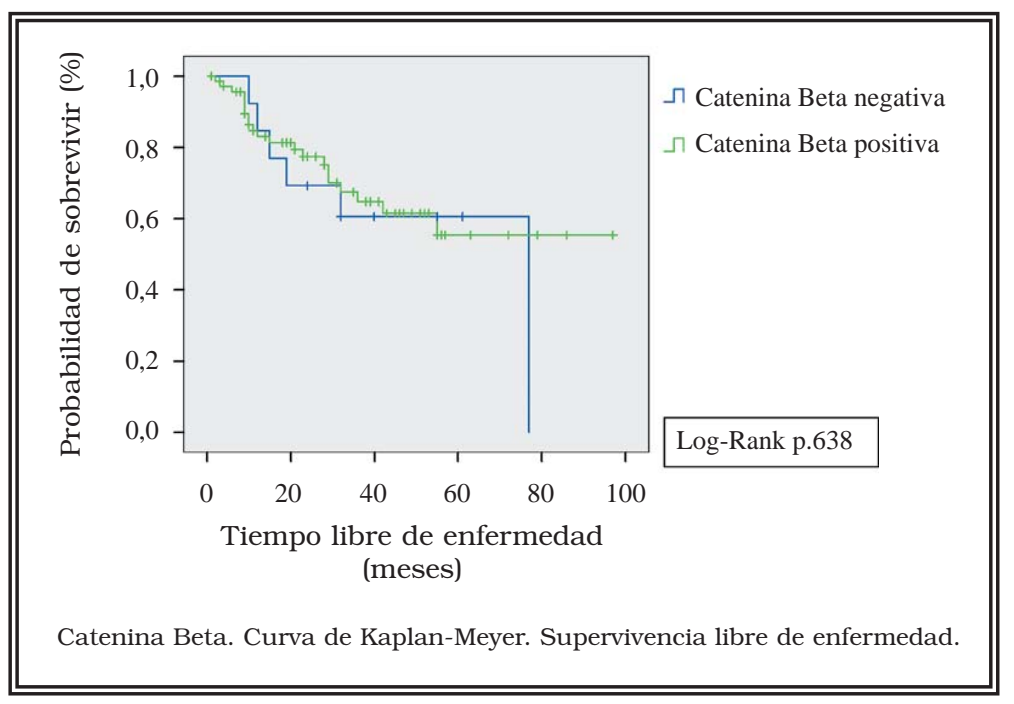

FIGURA 4. Inmunotinción para Cadherina E. (x 400)

\section{RESULTADOS}

Se analizan 88 tumores T1 con una mediana de tiempo libre de enfermedad de 36 meses.

El 58\% de la serie ha recibido tratamiento profiláctico con BCG $81 \mathrm{mg}$ semanal durante seis semanas.

En la Tabla 1 se muestran las características de los tumores, tanto en las variables "clásicas" como las de estudio.

Han recidivado el $31 \%$ de los tumores y progresado a estadio infiltrante el 3\%. Esta baja tasa de progresión no nos permite realizar un análisis pronóstico para la progresión tumoral.
En la Tabla 2 se muestra la correlación de las variables clásicas con las variables del estudio. La expresión de Ki-67 se correlaciona con grado $(\mathrm{p}, 002)$ y permeación linfática (p ,028). La multiplicidad tumoral con la falta de expresión de Cadherina E y Catenina Beta.

En la Tabla 3 se muestra la relación de las variables de estudio con la recidiva tumoral. Sólo la expresión de Ki-67 (p ,049) y la de Catenina Beta $(p, 039)$ alcanzan significación estadística.

Han recidivado el $38 \%$ de los que no han recibido tratamiento profiláctico frente al $28 \%$ de los que sí lo recibieron ( $p, 355)$.

En el estudio multivariante sólo la falta de expresión de Catenina Beta muestra tener valor pronóstico independiente para recidiva ( $\mathrm{p}, 049$; O.R:2,4-6,9).

La relación entre la expresión del inmunomarcador, la recidiva tumoral y el tratamiento profiláctico se muestra en la Tabla 4. Los tumores que expresan Catenina Beta $(p, 047)$ se benefician de tratamiento profiláctico.

El estudio de supervivencia libre de enfermedad se muestra en las Figuras 1 a la 4. Sólo la expresión de Catenina Beta y la Cadherina B alcanzan significación estadística ( $\mathrm{p}, 001$ y p ,034 respectivamente), siendo mayor la supervivencia cuando son positivas.

\section{DISCUSIÓN}

En nuestra serie sólo la expresión de Ki-67 ( $p, 049)$ y la de Catenina Beta ( $p$.039) se relacionan con la recidiva tumoral, recidivando con más frecuencia aquellos tumores que expresan el antígeno Ki-67 más del 10\% de sus células o aquellos que no expresan Catenina Beta. Está última es la que muestra valor pronóstico independiente de recidiva en el análisis multivariante, aunque la expresión de Ki-67 se sitúa muy cercana a la significación estadística ( $p$,082).

Respecto a la expresión de Ki-67 estos resultados se muestran acordes con los de la literatura consultada. La mayoría de los autores están de acuerdo en que la expresión de Ki-67 es un mar- 
Tabla 1

Características de la serie.

\begin{tabular}{lll}
\hline & Variable & \% \\
\hline Grado & G1 & 34 \\
& G2 & 46 \\
\multirow{4}{*}{ Linfático } & G3 & 20 \\
& Lx & 29 \\
Número & L0 & 67 \\
& Único & 4 \\
Tamaño & Múltiple & 66 \\
& & 34 \\
P53 & $<3$ cc & 38 \\
& $>3$ cc & 62 \\
Ki-67 & negativo & 84 \\
& positivo & 16 \\
Cadherina E & negativo & 63 \\
& positivo & 37 \\
Catenina Beta & positiva & 44 \\
& negativa & 56 \\
& positiva & 45 \\
& negativa & 55 \\
\hline
\end{tabular}

cador pronóstico independiente de recidiva, tanto a nivel internacional ${ }^{3,9,10,22,28,31-33}$ como nacional $^{16-18,35}$.

No hemos encontrado trabajos que relacionen expresión de Catenina Beta con recidiva tumoral, aunque sí con peor supervivencia y siempre asociada a la Cadherina E. En nuestra serie la falta de expresión de Catenina Beta tiene valor pronóstico independiente para recidiva tumoral. ¿Podría servir para indicar tratamiento profiláctico?.
En nuestra serie ni la Cadherina E ni la expresión de proteína p53 son factores pronósticos de recidiva, posiblemente por la baja tasa de progresión que presenta la misma. Como hemos visto en la literatura, la expresión de Cadherina E se asocia fundamentalmente a progresión tumo$\mathrm{ral}^{22,26}$. Igualmente muchos autores aceptan que la utilidad mayor de la p53 es predecir la progresión tumoral ${ }^{4-6,15,17,18}$.

Coincidiendo con la literatura tanto la falta de expresión de Cadherina E como de Catenina Beta se asocia a un menor tiempo libre de enfermedad, posiblemente porque la ausencia de expresión indica una mayor capacidad de diseminación y posible siembra intravesical, facilitando la recidiva tumoral.

Ninguno de los inmunomarcadores es útil para predecir la respuesta a las instilaciones con BCG. Estos resultados están en consonancia con los de la literatura, aunque ésta fundamentalmente se ha centrado en la relación entre p53 y BCG.

Sólo hemos encontrado menor recidiva, estadísticamente significativa en los tumores que sí expresan Catenina Beta y son tratados con BCG. Es decir aquellos tumores que a priori, según nuestro estudio, tienen menos probabilidades de recidivar.

La mayoría de los autores están de acuerdo en que la expresión de p53 previo al tratamiento no permite predecir la respuesta al mismo, aunque sí lo hace la expresión de p53 tras tratamiento con $\mathrm{BCG}^{6}$.

Tabla 2

Relación entre las variables clásicas y los inmunomarcadores. Se expresa en \%.

\begin{tabular}{|c|c|c|c|c|c|}
\hline \multicolumn{2}{|l|}{ Variables } & \multirow{2}{*}{$\frac{\text { P53 positivo }}{7}$} & \multirow{2}{*}{$\begin{array}{c}\text { Ki-67 positivo } \\
17\end{array}$} & \multirow{2}{*}{$\begin{array}{c}\text { Cadherina E negativo } \\
55\end{array}$} & \multirow{2}{*}{$\frac{\text { Catenina Beta negativa }}{50}$} \\
\hline Grado & G1 & & & & \\
\hline & G2 & 17 & 40 & 52 & 51 \\
\hline & G3 & 30 & 70 & 65 & 70 \\
\hline & Valor de $\mathrm{p}$ & n.s. & ,002 & n.s. & n.s. \\
\hline \multirow{4}{*}{ Linfático } & $\mathrm{Lx}$ & 12 & 53 & 56 & 56 \\
\hline & LO & 17 & 31 & 53 & 51 \\
\hline & L1 & 33 & 100 & 100 & 100 \\
\hline & Valor de $\mathrm{p}$ & n.s. & ,028 & n.s. & n.s. \\
\hline \multirow[t]{3}{*}{ Número } & Único & 21 & 35 & 27 & 42 \\
\hline & Múltiple & 7 & 45 & 72 & 69 \\
\hline & Valor de $\mathrm{p}$ & n.s. & n.s. & ,023 & ,046 \\
\hline \multirow[t]{3}{*}{ Volumen } & $<3 \mathrm{cc}$ & 12 & 36 & 43 & 60 \\
\hline & $>3 \mathrm{cc}$ & 18 & 43 & 51 & 61 \\
\hline & Valor de $\mathrm{p}$ & n.s. & n.s. & n.s. & n.s. \\
\hline
\end{tabular}


Tabla 3

Frecuencia de recidiva en relación con las variables

\begin{tabular}{lccc}
\hline & Variables & \% Recidiva & Valor de p \\
\hline Grado & G1 & 31 & n.s. \\
& G2 & 35 & \\
Linfático & Lx & 46 & n.s \\
& L0 & 29 & \\
Número & L1 & 33 & \\
& Unico & 31 & n.s. \\
Tamaño & Múltiple & 32 & \\
& $<3$ cc & 27 & n.s. \\
P53 & $>3$ cc & 34 & \\
& negativo & 29 & n.s. \\
Ki-67 & positivo & 43 & \\
& negativo & 22 & ,049 \\
Cadherina E & positivo & 43 & \\
& positiva & 26 & n.s. \\
Catenina Beta & negativa & 35 & \\
& positiva & 20 &, $\mathbf{0 3 9}$ \\
& negativa & 40 & \\
\hline
\end{tabular}

Tabla 4

Recidiva en relación al tratamiento y al status del marcador

\begin{tabular}{lcccc}
\hline (\%) & & $\begin{array}{c}\text { No } \\
\text { tratamiento }\end{array}$ & $\begin{array}{c}\text { BCG } \\
\text { p53 }\end{array}$ & $\begin{array}{c}\text { Valor de } \\
\mathbf{p}\end{array}$ \\
\hline \multirow{2}{*}{ Ki-67 } & Negativo & 37 & 26 & n.s. \\
& Positivo & 43 & 43 & \\
Cadherina E & Negativo & 28 & 23 & n.s. \\
& Positivo & 50 & 36 & \\
Catenina Beta & Negativo & 33 & 36 &, 059 \\
& Positivo & 45 & 18 & \\
& Negativo & 44 & 39 &, $\mathbf{0 4 7}$ \\
\hline
\end{tabular}

Existen pocas publicaciones que relacionen Ki-67 con BCG, con resultados dispares y no hemos encontrado ninguna que relacione las moléculas de adhesión con la inmunoterapia con BCG.

\section{CONCLUSIONES}

- Los marcadores más útiles para predecir recidiva tumoral son Ki-67 y Catenina Beta, recidivando más cuando son Ki-67 positivos y/o Catenina Beta negativos.

- Sólo la Catenina Beta muestra un valor independiente para recidiva tumoral en el estudio multivariante, si bien la expresión de Ki-67 se encuentra muy cercana $(p, 082)$.
- Tanto la falta de expresión de Catenina Beta como de Cadherina $\mathrm{E}$ se asocian a un menor tiempo libre de recidiva.

- Los tumores tratados con BCG que sí expresan Catenina Beta tienen un número de recidivas menor. Dado que en nuestro estudio estos tumores son los que menos recidivan, creemos que la expresión o no de Catenina Beta no debe influir en la decisión de utilizar BCG.

\section{REFERENCIAS}

1. Esrig D, Elmaijan D, Groshen SJ, Freeman JA, Stein JP, Chen SC et al. Accumulation of nuclear p53 and tumour progression in bladder cancer. N Engl J Med. 1994;331 (19): 1259-1264.

2. Su JS, Arima K, Hasegawa M, Franco OE, Yanagawa M, Sugimura $\mathrm{Y}$ et al. Proliferative status is a risk index for recurrence in primary superficial (pTa/T1) low-grade urothelial bladder carcinoma. Hinyokika Kiyo. 2003;49(11): 649-658.

3. Saint F, Le Frere Bedla MA, Quintela R. Pretreatment p53 nuclear overexpression as a prognositc marker in superficial bladder cancer treated with bacillus calmette-guerin (BCG). Eur Urol. 2004;45(4):475-482.

4. Serth J, Kuczyk C, Bokemeyer C, Hervatin C, Nafe R, Tan HK et al. P53 inmunohistochemistry as an independent prognostic factor for superficial transitional cell carcinoma of the bladder. British J Cancer 1995;71(1):201-205.

5. Sarkis AS, Dalbagni G, Cordon Cardo C, Zhang ZF, Sheinfeld J, Fair WR et al. Nuclear overexpression of p53 protein in transitional cell bladder carcinoma: a marker for disease progression. J Natl Cancer Inst. 1993;85(1):53-59.

6. Lacombe L, Dalbagni G, Zhang ZF, Cordon Cardo C, Fair WR, Herr HW et al. Overexpression of p53 protein in a high risk population of patients with superficial bladder cancer before and after bacillus Calmette-Guerin therapy: correlation to clinical outcome. J Clin Oncol. 1996;14(10):26462652.

7. Ramos Soler D, Navarro Fos S, Villamon Fort R. Estudio comparativo de la expresión de p53, Bcl-2 y C-erb-2 en neoplasias papilares vesicales de bajo grado. Arch Esp Urol. 2003;56(3):277-285

8. Pfister C, Moore L, Allard P, Larue H, Lacombe L, Tetu B et al. Predictive value of cell cycle markers p53, MDM2, p21, and Ki-67 in superficial bladder tumor recurrence. Clin Cancer Res. 1999;5(12):4079-4084

9. Stavropoulos NE, Filiadis I, Ioachim E, Hastazeris K, Tsimaris I, Kalogeras D et al. Prognostic significance of p53, bcl-2 and Ki-67 in high risk superficial bladder cancer. Anticancer Res. 2002;22(6B):3759-3764.

10. Shariat SF, Weizer AZ, Green A, Laucirica R, Frolov A, Wheeler TM et al. Prognostic value of P53 nuclear accumulation and histopathologic features in T1 transitional cell carcinoma of the urinary bladder. Urology. 2000;56(5): 735-740.

11. Burkhard FC, Markwalder R, Thalmann GN, Studer UE. Inmunohistochemical determination of p53 overexpression. An easy and readily available method to identify progression in superficial bladder cancer?. Urol Res. 1997; 25(suppl 1):31-35. 
12. Liukonnen T, Rajala P, Raitanen M, Rintala E, Kaasinen E, Lipponen P. Prognostic value of Mib-1 score, p53 ,EGFr, mitotic index and papillary status in primary superficial (stage pTa/t1) bladder cancer: a prospective comparative study. Eur Urol. 1999;36(5):393-400.

13. Masters JRW, Vani UD, Grigor KM, Griffiths GO, Crook A, Parmar MK et al. Can p53 staining be used to identify patients with agressive superficial bladder cancerr? J Pathol. 2003;200(1):74-81.

14. Vet JA, Bringuier PP, Schaafsma HE. Comparison of p53 protein overexpression with p53 mutation in bladder cancer: clinical and biologic aspects. Lab Inves. 1995;73(6):837-843.

15. Menéndez López V, Molina Porto R, Alcaraz Asensio A, Gutiérrez del Pozo R, Alcover García JA. Relationship between tumor recurrence and the expression of the p53 gene in primary superficial transitional carcinoma of the bladder. Actas Urol Esp. 2000;24(7):530-535.

16. San Miguel Fraile P, Antón Badiola I, Ortiz Rey JA, Álvarez Alvarez C, Fernández Costas A, Lago Fernández $\mathrm{M}$ et al. Estudio comparativo de la expresión de p53, Ki-67, bcl-2 y CK20 en el carcinoma transicional superficial de vejiga: correlación con la recurrencia, grado histológico y estadio clínico. Actas Urol Esp. 2003;27(8):587-593.

17. Moyano Calvo JL, De Miguel Rodríguez M, Ortiz Gámiz A, Poyato Galan JM, Sánchez Sánchez E, Blanco Palenciano $\mathrm{E}$ et al. Determinación de ploidía de ADN mediante citometría de flujo, índice Ki-67, sobreexpresión de proteína p53 en 121 carcinomas superficiales de vejiga T1. Estudio retrospectivo. Correlación con las variables clásicas. Actas Urol Esp. 2000;24(10):785-795.

18. Rodríguez Alonso A, Fernández Pita S, González Carrero J, Nogueira March Jl. Análisis multivariado de recidiva y progresión en el carcinoma de células transicionales de vejiga en estadio T1. Valor pronóstico de p53 y Ki-67. Actas Urol Esp. 2003;27(2):132-141.

19. Bustos Castañón L, Sánchez Merino JM, Picallo Sánchez A, Gelabert Mas A. Expresión del receptor del factor de crecimiento epidérmico y de la proteína p53 en el cáncer superficial de vejiga. Arch Esp Urol. 2001;54(1):13-21.

20. Moreno Sierra J, López García Asenjo JA, Redondo González E. Utilidad de la oncoproteína p53-IHQ en el seguimiento del carcinoma vesical: estudio a 5 años. Arch Esp Urol. 1999;52(8):840-848.

21. Rebassa Llull M, Gutiérrez Sanz-Gadea C, Muñoz Vélez D, Esteva Bauza M, Gómez Bellvert C, Mus Malleu A et al. Expression of protein p53 in superficial transitional carcinoma of the bladder with differing course. Arch Esp Urol. 1999;52(10): 1061-1064.

22. García Del Muro X, Torregrosa A, Munoz J, Castellsague $\mathrm{X}$, Condom E, Vigues $\mathrm{F}$ et al. Prognostic value of the expression of E-cadherin and Beta-catenin in bladder cancer. Eur J Cancer. 2000;36(3):357-362.

23. Syrigos Kn, Krausz T, Waxman J, Pandha H, RowlinsonBusza G, Verne $\mathrm{J}$ et al. E-cadherin expression in bladder cancer using formalin-fixed, paraffin-embedded tissues: correlation with histopathological grade, tumour stage and survival. Int J Cancer. 1995;64(6):367-370.

24. Nakopoulou L, Zervas A, Gakiopoulou-Givalou H, Constantinides C, Doumanis G, Davaris P et al. Prognostic value of $\mathrm{E}$ - cadherine, Beta-catenin, P120 cten in patients with transitional cell bladder cancer. Anticancer Res 2000 (6B);20:4571-4578.
25. Lipponen PK, Eskelinen MJ. Reduced expression of E-cadherin is related to invasive disease and frequent recurrence in bladder cancer. J Cancer Res Clin Oncol. 1995;121 (1):303-308.

26. Shariat SF, Pahlavan S, Baseman AG, Brown RM, Green $\mathrm{AE}$, Wheeler $\mathrm{TM}$ et al. E-cadherin expression predicts clinical outcome in carcinoma in situ of the urinary bladder. Urology 2001;57(1):60-65.

27. Thievsen I, Seifert HH, Swiatkoswski S, Fiori AR, Schulz WA. E-cadherin involved in inactivation of WNT/Beta-catenine signalling in urothelial carcinoma and normal urothelial cells. Br J Cancer 2003;88(12):1932-1938.

28. Shimazui T, Schalken JA, Giroldi LA, Jansen CF, Akaza H, Koiso K et al. Prognostic value of cadherin-associated molecules (alpha-, Beta-, and gamma-catenins and p120 cas) in bladder tumors. Cancer Res. 1996;56(18):4154-4158.

29. De Chirife A-M, Giménez L, Marino L, Celeste F. Estudio de la fracción de crecimiento en el carcinoma transicional de vejiga. Arch Esp de Urol. 1993;46(1):7-11.

30. Santos L, Amaro T, Costa C, Pereira S, Bento MJ, Lopes P et al. Ki-67 index enhances the prognostic accuracy of the urothelial superficial bladder carcinoma risk group classification. Int J Cancer. 2003;105(2):267-272.

31. Asakura T, Takano Y, Iki M, Suwa Y, Noguchi S, Kubota Y et al. Prognostic value of Ki-67 for recurrence and progresión of superificial bladder cancer. J Urol. 1997;158(2):385-388.

32. Blanchet P, Droupy S, Eschwege P, Viellefond A, Paradis V, Pichon MF et al. Prospective evaluation of Ki-67 labeling in predicting the recurrence and progression of superficial bladder transitional cell carcinoma. Eur Urol. 2001;40(2): 169-175.

33. Gontero P, Casetta G, Zitella A Ballario R, Pacchioni D, Magnani C et al. Evaluation of P53 protein overexpression, Ki67 proliferative activity and mitotic index as markers of tumour recurrence in superficial transitional cell carcinoma of the bladder. Eur Urol. 2000;38(3):287-296.

34. López-Beltrán A, Luque RJ, Álvarez-Kindelan J, Quintero A, Merlo F, Requena MJ et al. Prognostic factors in survival of patients with stage Ta and T1 bladder urothelial tumors. The role of G1-S modulators (p53, p21Waf1, p27Kip1, Cyclin D1 and Cyclin D3), proliferation index and clinicopathologic parameters. Am J Clin Pathol. 2004;122 (3):444-452.

35. Moyano Calvo JL, De Miguel Rodríguez M, Poyato Galan JM, Ortiz Gámiz A, Molina Carranz A, Zerpa Railey J et al. Determinación de ploidía de ADN mediante citometría de flujo, índice Ki-67, sobreexpresión de proteína p53 en 121 carcinomas superficiales de vejiga T1. Estudio retrospectivo. $2^{\text {a }}$ Parte: Valor pronóstico y utilidad en la indicación de tratamiento profiláctico con BCG. Actas Urol Esp. 2001;25 (1):32-45.

Dr. J.L. Moyano Calvo

Servicio de Urología. Hospital Virgen Macarena

Avda. Dr. Fedriani, s/n

41071 Sevilla

E-mail: joseluismoyano@ya.com

(Trabajo recibido el 6 de septiembre de 2005) 